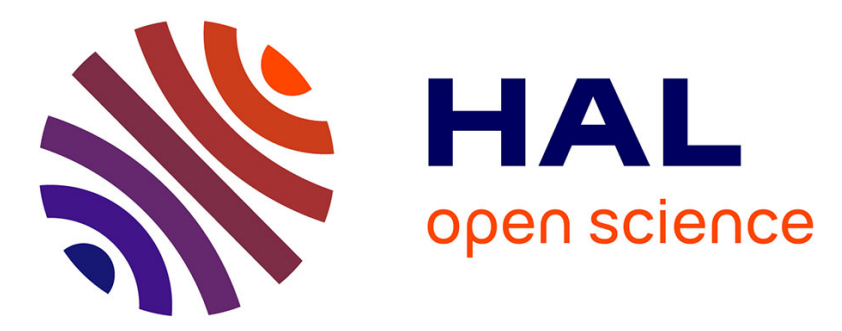

\title{
Product Development in Collaborative Networks - An Expert View on Current Challenges and Future Trends
}

Wei Min Wang, Pascal Lünnemann, Sebastian Neumeyer, Haygazun Hayka, Rainer Stark

\section{- To cite this version:}

Wei Min Wang, Pascal Lünnemann, Sebastian Neumeyer, Haygazun Hayka, Rainer Stark. Product Development in Collaborative Networks - An Expert View on Current Challenges and Future Trends. 17th Working Conference on Virtual Enterprises (PRO-VE), Oct 2016, Porto, Portugal. pp.302-312, 10.1007/978-3-319-45390-3_26. hal-01614619

\author{
HAL Id: hal-01614619 \\ https://hal.inria.fr/hal-01614619
}

Submitted on 11 Oct 2017

HAL is a multi-disciplinary open access archive for the deposit and dissemination of scientific research documents, whether they are published or not. The documents may come from teaching and research institutions in France or abroad, or from public or private research centers.
L'archive ouverte pluridisciplinaire HAL, est destinée au dépôt et à la diffusion de documents scientifiques de niveau recherche, publiés ou non, émanant des établissements d'enseignement et de recherche français ou étrangers, des laboratoires publics ou privés.

\section{(c)(1)}

Distributed under a Creative Commons Attribution| 4.0 International License 


\title{
Product Development in Collaborative Networks - An Expert View on Current Challenges and Future Trends
}

\author{
Wei Min Wang ${ }^{1}$, Pascal Lünnemann ${ }^{2}$, Sebastian Neumeyer ${ }^{2}$, Haygazun Hayka ${ }^{2}$ \\ Rainer Stark ${ }^{1,2}$ \\ ${ }^{1}$ Department for Machine Tools and Factory Management, Technische Universität Berlin, \\ Pascalstr. 8-9, 10587 Berlin, Germany \\ w.wang@tu-berlin.de \\ ${ }^{2}$ Department forVirtual Product Creation, Fraunhofer-Institute for Production Systems and \\ Design Technology, Pascalstr. 8-9, 10587 Berlin, Germany \\ \{pascal.luennemann, sebastian.neumeyer, haygazun.hayka \\ rainer.stark\}@ipk.fraunhofer.de
}

\begin{abstract}
Cross-enterprise collaboration in product development has become a common practice in industry. Companies increasingly depend on collaborations with networks of partners from different tiers of value creation, various geographic locations and even from outside of their own sector. Moreover, there is a growing tendency to involve parties from later stages of the product's lifecycle in the product development phase. To face the challenges of the increasing complexity of collaborative product development a holistic approach of Product Lifecycle Management (PLM) has evolved. Although methods, processes and IT-systems of PLM proved to be beneficial in supporting the internal collaboration of industrial companies, they seem to have only little effect in the context of cross-company collaboration. Reasons and solution approaches for that were evaluated in an expert study with 40 experts from industry and academia. Results from that study and practical implications are presented in this paper.
\end{abstract}

Keywords: Product Development in Collaborative Networks; Collaborative Engineering, Product Lifecycle Management, Industrial Collaboration Practices, Expert Study, Trends of Collaborative Engineering

\section{Introduction}

The development of modern products often requires interdisciplinary collaboration of several companies $[1,2]$. This is partly a consequence of the increasing product complexity that is driven by trends such as Industry 4.0 and Product Service Systems (PSS) [2]. For example, cyber-physical products may be offered with accompanying services or even completely as a PSS. In both cases knowledge from various domains have to be combined. Furthermore, the expanding scope of product functions, their maintenance and repair often requires the knowledge of several companies to be merged to develop the product [1]. Hence, interdisciplinary and cross-company collaboration in product development has become a common practice in industry, causing companies to increasingly depend on collaborations with networks of partners 
from different tiers of value creation, various geographic locations and even from outside of their own sector [3]. Moreover, there is a growing tendency to involve parties from later stages of the product's lifecycle in the product development phase. The transformation of the enterprise-wide product development also requires an adaptation of the working environment in terms of tools, IT systems, technical infrastructure, information and data models, processes and organizational structures.

To meet these demands while achieving short development cycles, cooperation between enterprises will become even more intense. This and the widely dispersed allocation of the necessary expertise are driving the conversion of the former coordinated product development into a collaborative product development. To identify essential aspects of innovations and obstacles regarding planning and implementation of measures to realize this change, an expert study on product development in collaborative networks was conducted by the Fraunhofer Institute for Production Systems and Design Technology (Fraunhofer IPK) in cooperation with the CONTACT Software GmbH and the Association of German Engineers (VDI). In this study 40 experts from industry and academia with relevant experience in the field of Product Data Management (PDM) / PLM and engineering collaboration were asked to share their expertise on current challenges and future trends. The results of these interviews give insights to relevant drivers for cross-company collaboration from both the industrial and the academic perspective and combines empirical knowledge from industry and innovations from PLM system vendors, consultancies, research institutes and universities. Based on these insights, best- and worst-practices from industry and strengths and weaknesses of current collaboration approaches are discussed. Finally, expectations regarding collaboration processes, respective IT-tools and strategic goals are derived, especially with regard to PDM and PLM.

Comparing to other survey in the field of engineering collaboration this study focused on the value creating engineering activities performed by people in a multienterprise environment, their coordination through processes and benefits of used IT tools. While the general motivation for collaboration is comparable to existing studies (e.g. [4] although this study focuses more on the social aspect of collaboration). While several current studies analyze the collaborative software development (e.g. the controlling and tools $[5,6])$ the presented study focuses mechatronic and cyber-physical systems as multi-domain products integrating different areas of product development.

Overall, it can detect that the collaborative product development is facing multifaceted challenges, in particular:

The change in the working situation, the increase of CPS, advancing globalization and related performance in data deployments and integration challenge the collaboration. As well as the coordination of very large and distributed development networks with nowadays worse transparency in the procedures.

And the optimizations of the provided it-support, in particular in their alignment with the development activities and processes, as well as the data security, data availability and user friendly interface design need to be addressed. Especially these challenges are taken into consideration in the context of this paper. 


\section{Study Design and Implementation}

\subsection{Definition of Collaboration in Context of Product Development}

There are numerous definitions of collaboration depending on the context of its application. Based on the definition of Lu et. al [7] this study regards collaboration in context of product development as an collective endeavor that "requires a team of individuals to work on tasks that not only have shared resources and shared outcomes, but, most importantly, a shared common goal". He distinguishes collaboration from coordination and cooperation as lower levels of collective endeavors. While coordination only requires sharing of resources to fulfil a collective task, cooperation also includes sharing of outcomes, but in both cases tasks designed to be disjunctive and are carried out independently. Whereas, collaboration explicitly requires combination of individual skills and collective efforts to fulfill tasks in order to reach a common goal. Therefore, organizational boundaries are neglected in favor of continuous exchanges of knowledge, information and resources [7]. Steinheider [8] further characterizes collaboration by the dimensions Coordination, Communication and Knowledge integration, each describing characteristics regarding one specific aspect of collaboration (see Fig. 1). As this study also evaluates IT-supported processes, the fourth dimension Information logistics was introduced, to provide a better understanding of information distribution within the product development process and throughout the lifecycle.

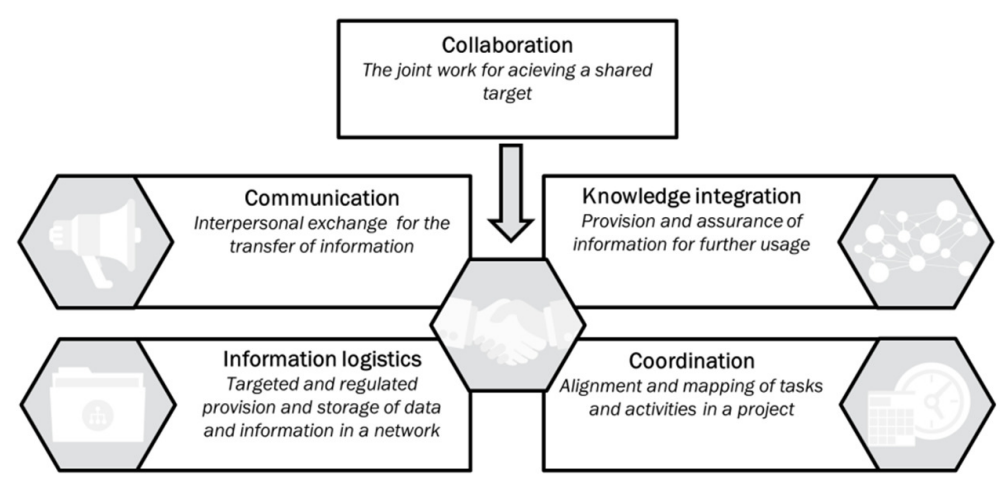

Fig. 1. Four dimensions of collaboration regarded in this study

\subsection{Structure of the Study}

The interviews were based on a standardized and structured questionnaire comprising closed and open questions, grouped in four sections (see Fig. 2). The first section focused on the evaluation of the current working situation and expected changes in collaborative product development with regard to the four dimensions mentioned before. In the second section, current practice and prospective future forms of 
cooperation are considered. Especially, the changing engineering environment and the related coordination aspects are considered there. In the third section, actual industrial practices and used tools are analyzed and best- and worst-practices are examined. In the fourth section, requirements regarding collaborative product development in the future are collected. These questions aim at the evaluation of both, current technological trends and organizational forms. Based on this structure, the study captures the current situation of collaboration in product development and shows a potential path for its development in the medium-term (i.e. in the coming five to ten years) from the surveyed experts' perspectives.

As part of the analysis of the responses recorded and protocolled conversations were analyzed on clusters and summarized in qualitative statements. According to the relatively small sample the responses were rated primarily qualitative and not quantitative. Even so in statistical reports for prioritizing statements equivalent weights were made (for example, placement weighting 1 to 5 ) and were taken in consideration accordingly in the qualitative analysis.

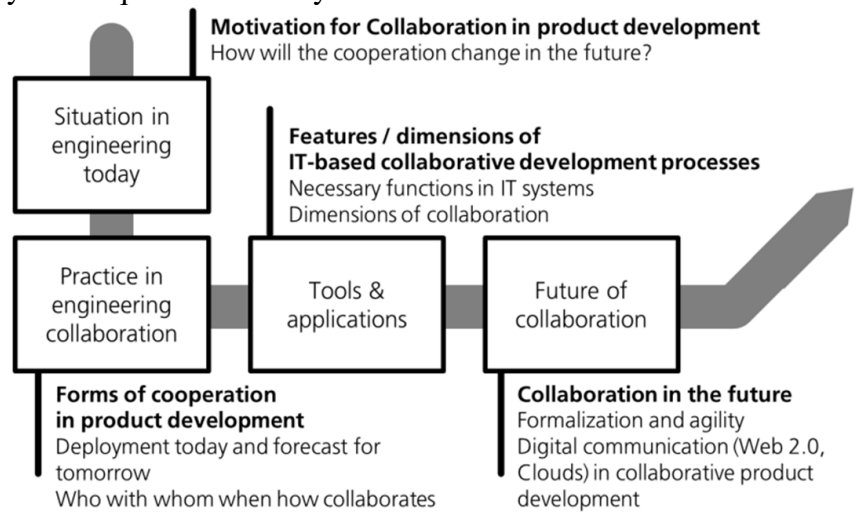

Fig. 2. General structure and core questions of the study

In total, 40 experts were interviewed using the structured questionnaire. The interviews were mostly conducted through phone calls and web-conferencing and took about one to two hours. In order to ensure a balanced mixture of practical industrial experience and innovative research, about one third of the interviewees are acquired from research institutes. Hereby, both an image of the current challenges and needs, as well as the currently under consideration innovations in research can be determined.

Two-thirds of the participants comprise representatives of the automotive industry, plant construction, consulting, aerospace and rail transportation (see Fig. 3a). With the aim of a possible heterogeneous and comprehensive picture of the challenges in enterprise-wide development, different roles were interviewed to get into the business. The models range from the experts in process management and information technology, corporate research and development departments (product-related), project managers and managers in departments, division and company boards. That way, both the practical work of community development as well as the strategic perspective of cooperation could be considered. Half of the participants from industry hold executive positions in their companies. With regard to the academic participants, the surveyed 
professors were counted analogously (see Fig. 3b). About half of the industrial participants work in OEMs, the other half in first-tier supplier companies.

a)

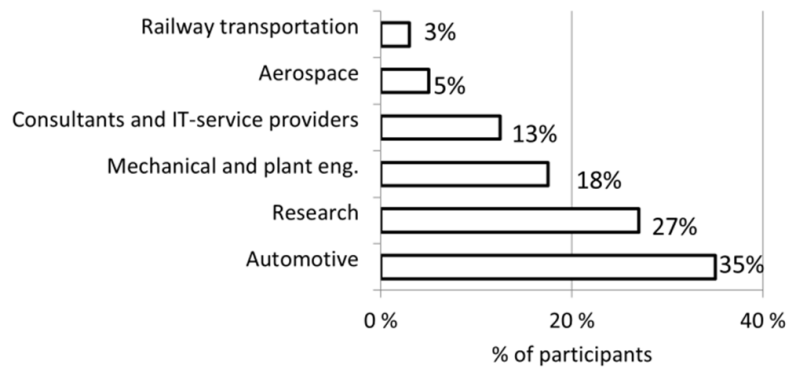

b)

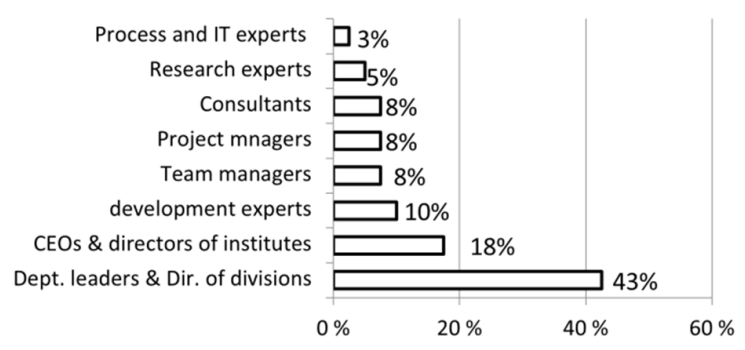

Fig. 3. a) Branches of experts, b) Positions held by the experts

\section{$3 \quad$ Findings}

\subsection{Changes in Collaboration Practices}

The increasing scope of product functions, new business models including product related services (e.g. PSS), the integration of software systems (e.g. user assistant systems) and communications to peripheral infrastructure (e.g. for condition monitoring) often require skills and expertise beyond the capabilities of one single company. In this respect, cross-enterprise and cross-sector collaborations are often seen as a way to acquire the necessary expertise [1]. This significance of this well-known driver is also confirmed by the answers of the surveyed experts. The acquisition of know-how, which is not or not sufficiently available in the own company, was ranked first by roughly $30 \%$ of the respondents. The second main driver is Cost reduction that refers to both, outsourcing in low-wage countries as well as the national subcontracting of specialized engineering services.

With regard to the duration of collaboration, the experts expect that the current timeframes of joint development projects will rather remain in a similar scope or even decrease. This reflects the decreasing duration of development projects per se, which in turn results from the ever more rapid innovation-cycles. At the same time, the experts expect that the duration of partnerships between the companies will increase or at least 
remain stable. This opposing development is explained as consequence of overheads invested. As stated by the respondents, it is anticipated that long-term partnerships allow for "smoother" collaboration processes as mutual trust between involved engineers can be established and organizational structured harmonized.

Concerning the total number of partners within collaboration, two opposing developments are anticipated by the experts. On the one hand, there is a trend of consolidation of small and medium sized suppliers into larger system-suppliers (e.g. through merger and acquisition). On the other hand, a growing number of small and highly specialized component suppliers are entering the market. Which effect will outweigh depends could not be determined generally, but will depend on the tier-level and the industrial sector. However, more than the half of the experts is expecting a general increase in the amount of partners. Only twenty percent are assuming a decrease. Especially, experts from companies of second-tier suppliers / developers expect a general increase in consortia sizes.

In parallel, the experts also describe a globalization of their development activities, as local development centres for localized modules are established often near- or onsite of OEMs to compete with other global acting companies. As a consequence, intercultural work has to be increasingly considered in engineering collaboration. From the experts' viewpoint, the current mode of collaboration, where partners are only involved for the detailed engineering, will be extended to other lifecycle phases such as concept design, market analysis or later phases such as production planning and manufacturing as well as supporting services.

\subsection{People are the Success Factors of Collaborations}

As defined by Lu et. al [7], collaboration is the highest form of collective endeavour requiring the sharing and alignment of resources and goals, and the integration of knowledge. As it is also fundamentally a social process [9], the success of collaboration often highly depends on the involved individuals and their social relationships [10]. In the context of cross-enterprise collaboration, this aspect is even more important. Firstly, a huge variety of individuals with divers cultural and professional backgrounds will come together when organizations from different industrial sectors and geographic locations engage in collaboration. Secondly, there is an additional level of organizational culture and habits, which may influence the collaboration behaviour of individual persons [9]. Hence, the individuals engaged in collaborative projects are the key success factors. Still, the development of tools and IT-system to support collaboration often tends to focus on technical requirements. This aspect was also stressed by the experts surveyed in this study. Various statements pointed out that the human is getting increasingly "lost" in the process of digitalization. One expert stated: "The IT-support for collaboration have reached a good maturity level, but the human factor within such processes have to be regarded. It is crucial to include direct communication on personal level to facilitate establishing a common base and building of mutual trust. " "Another respondent from industry even said that "it has been forgotten, that every IT-system is used by humans". He pointed out, that the

1 All citations are analogous translations of the statements made in German 


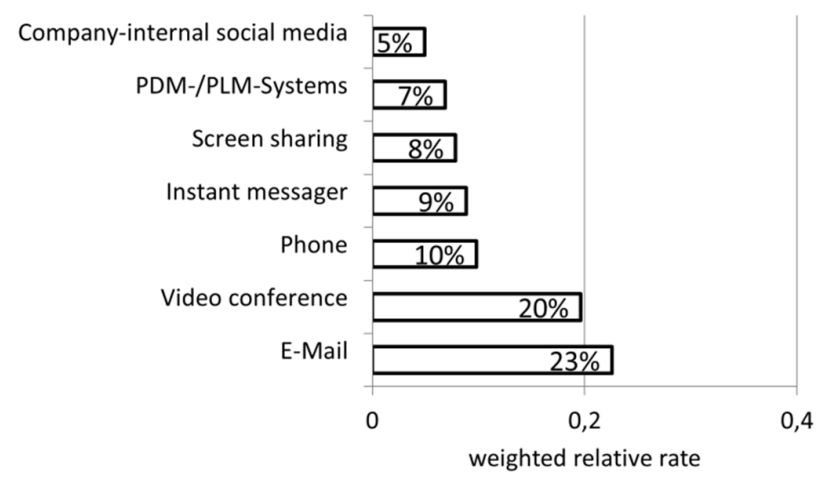

Fig. 4. Tools used for communication in industrial practice

development of tools is often heavily dominated by technical requirements, while the human factor is totally neglected. In his opinion, this kind of focus led to "disastrous" user interfaces in all current PDM systems.

As confirmation of these statements, the summarized responses showed that personal discussions are the preferred best practice (20\% of total answers), followed by the realization if transparency ( $11 \%$ of total answers) and communicative or spontaneous employees. Also the usage of multimedia as well as a frequent communication and a common language are success factors. The usage of email as main communication channel has been identified in a previous study conducted by Müller et. al in 2013 [11], and could be confirmed again in the current study ( $23 \%$ of answers). In addition, video conferences have been mentioned (20\%), while the Phone as well as instant messaging tools, screen sharing and PDM-/PLM-Solutions for communication have been mentioned with $10 \%$ or less(see Fig. 4).

The coordination of an engineering collaboration is due to different organizations, their different cultures and processes, always a challenge in projects. The previous study [11] revealed in this regard that coordination and communication are covering more than twenty percent of the working time of design engineers. This was also confirmed by the experts in the current study. As best practice for coordination, a shared comprehension of processes (18\%) as well as taking responsibility (11\%) and scheduled deliverables (10\%) have been highlighted by experts. In contrast, coordination is inhibited from the experts' point of view by unsuitable IT-solutions, bad leadership and missing process comprehension. These results again shows that the persons involved in the collaborative activities are of integral meaning to it's success. 
Preferred IT-solutions in context of coordination are project management tools with nearly one third of mentioned answers (29\%). PDM- and PLM-solutions (15\%), workflow management $(15 \%)$ and project management functionalities in PLMsolutions (13\%) turned out to be less important as expected (see Fig. 5).

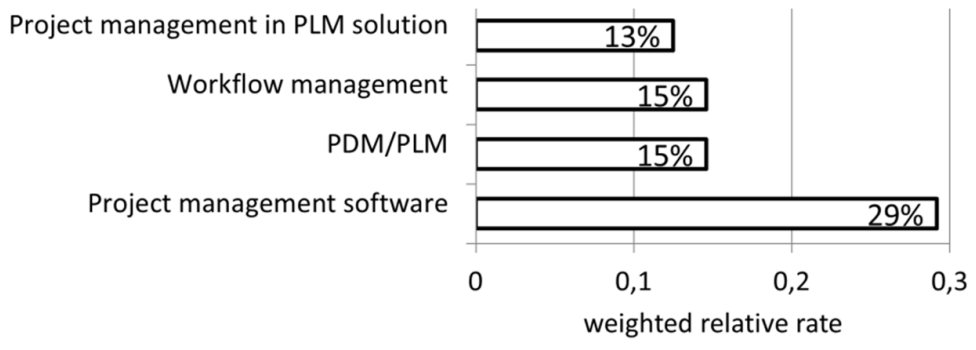

Fig. 5. Tools used for coordination in industrial practice

Beside coordination and communication, the information logistics in engineering collaboration need to be considered for a holistic view. To provide the engineer with the right information at the right time is the most important factor from the experts' viewpoint (16\% of answers). This aspect is followed by proper access privilege management $(11 \%)$, availability of the information $(9 \%)$ and clear specification $(7 \%)$ as best practice in information logistics (see Fig. 6).

Security concerns as well as unclear interfaces, unreliable IT-systems and missing information within an IT-system are show stoppers in this dimension. These IT-systems are in majority PDM-/PLM-Solutions (33\%), while only $6 \%$ of the experts confirmed that direct partner integration in PDM-/PLM-solutions is given. Commonly used solutions for data exchange are dedicated Team Data Management (TDM) systems. Cloud-solutions were rarely mentioned in the answers $(7 \%)$ and are hence, considered exceptions in information logistics (see Fig. 7a).

The knowledge integration as the last building block is understood as methodological and procedural definition of the mode of operation and the securing of gained knowledge of an engineering collaboration. However, the current activities are focussing on documentation and transfer of explicit knowledge. Therefore, handbooks (14\%), guidelines (12\%) and IT-systems (12\%) are used. The use of Wikis for continuous documentation of group knowledge, the capturing of abstracted knowledge

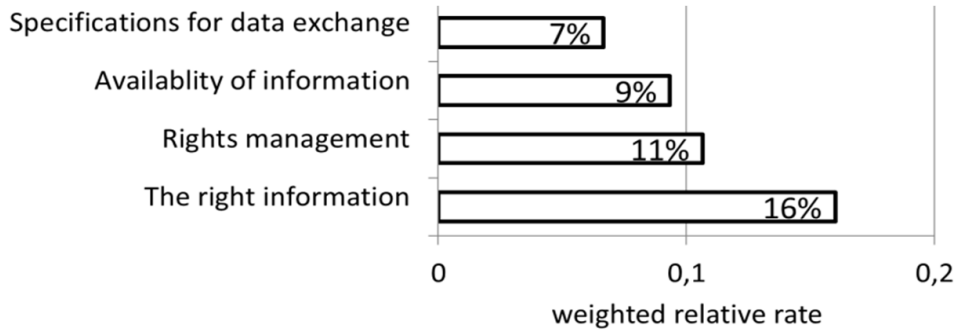

Fig. 6. Best practices in information logistics 
as well as lessons learned were only mentioned peripheral (7\% each). In this regard, the human factor proves to be a major hindrance, as one essential reason for insufficient knowledge integration is the lack of personal motivation share or provide knowledge. This is mainly due to a low acceptance of that activity per se (17\%), resulting partly from concerns about becoming easier replaceable and partly from a lack of defined processes. This in turn results in inadequate documentation and useless efforts. A further obstacle for knowledge integration is that the value of knowledge management is often not or not clearly defined in the working context of engineers $(6 \%)$. Hence, it is rather perceived as an overhead than as essential value creation activity.

Integration of partners in PDM/PLM

Cloud-based solutions

a)

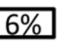

b)
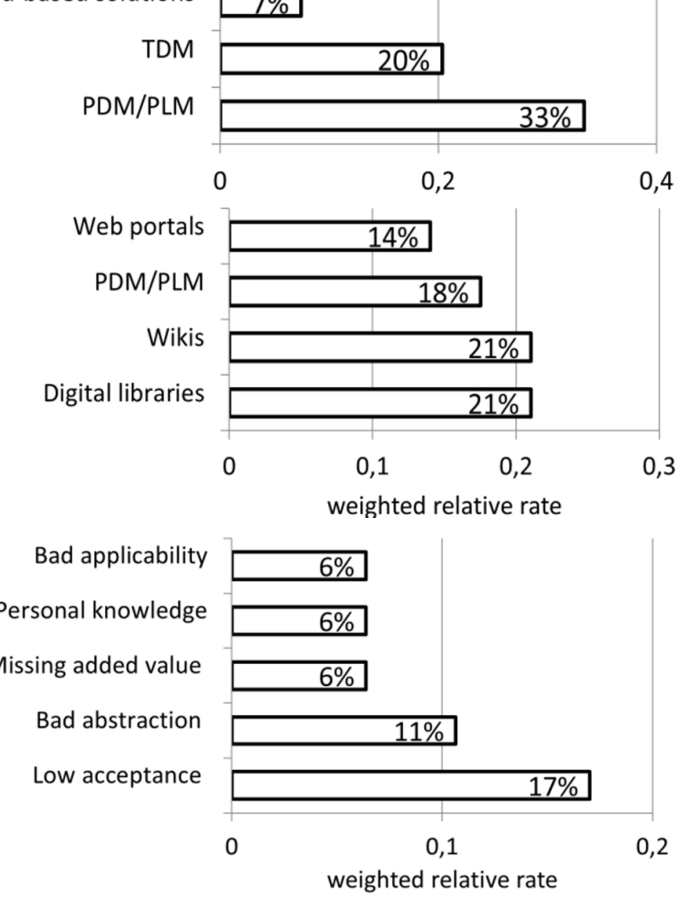

Fig. 7. a) Tools used in information logistics, b) Tools used in knowledge integration c) Worst cases in knowledge integration 


\section{Conclusion}

The current situation in engineering collaboration is characterized by a wide variety of tools, interfaces, communication channels and process definitions. Due to security reasons, companies usually do not allow partners to access their central data sources, therefore the internal project members have to spend high efforts to search, extract and transfer data to portals so partners and suppliers can access them.

Partners have to deal with the challenge, that each customer provides different interfaces, individual processes and rules for the execution of collaboration. Information, data and processes need to be persistent though all the layers of developers, which hardly can be achieved by a high amount of interfaces and formats. While today engineers are already overwhelmed by the existing process instructions.

In addition, neither the currently implemented PLM-solutions provide the necessary flexibility and openness, so individual as well as different approaches for partner integration can be achieved. Nor the necessary human-to-human communication is adequately supported.

In consequence, collaboration needs to be planned and executed in a more holistic way and not treated only as a technical solution or a process definition. Due to the finding, that collaboration will extend, the number of partners will increase and the integration of non-sector specific partners is expected, collaboration need to be treated under multidimensional aspects with special emphasis on the human claims. Only by covering processes and organizations, tools and IT-systems as well as information and data models aligned with activities performed by people, the engineering collaboration will be a success.

With regard to the PLM-approach, the holistic consideration of all these aspects has to be covered to achieve a successful collaboration. Last but not least the most important element of collaboration has been mentioned: only with individual humans, who are motivated, communicative, upright and flexible with different situations, collaboration will finally succeed.

Acknowledgments. We thank all participants of the study for their contribution and support. Only by their individual commitment and their willingness to answer our questions patiently, even at early and late hours, this study could gained such in-depth insights about the present and future of collaboration in product development. We also want to thank Ms. Helena Rott (Fraunhofer IPK) and Ms. Barbara Scholvin (CONTACT Software $\mathrm{GmbH}$ ) for their persistent contribution to the text editing and creation of graphics. Finally, we thank Dr. Heinz Bedenbender (VDI-GPP) and Ms. Sophia Rogge (TU Berlin) for proofreading. 


\section{A Appendix}

Structure of the questionnaire and content summary:

1. Introduction:

flow and content of the interview

2. Demographics:

Information about the person, the company and the role

3. Drivers for cooperation:

Key drivers in prioritization

4. Forms of cooperation:

(Industry:) What forms are used, as these affect the daily life, as they affect the IT support

(All:) changes in collaboration situation (duration, form, size, accompanying processes, strategy and partnership)

5. Features of collaborative development processes:

guarantee of success, show-stopper and IT support in the dimensions of communication, coordination, information logistics and knowledge integration.

IT support in the areas of requirements management, risk management, project management, change management, BOM maintenance, data exchange

6. Cooperation in the future:

process orientation and its flexibility, transparency in the collaboration, language barriers, technological trends, Clouds, mobile devices

7. Statement of the interviewee 


\section{References}

1. Krause F, Hayka H, Pasewaldt B. Efficient product data sharing in collaboration life cycles. In: Advances in Design. London: Springer; 2006. p. 365-75.

2. Stöckert H. Fehlervermeidung an Schnittstellenprozessen der verteilten Produktentwicklung.

3. Hayka H, Staub G, Thamburaj V, Zhang Q. Kooperationsplattform fur mechatronische Produktentstehung. ZWF: Zeitschrift für Wirtschaftlichen Fabrikbetrieb. 2008;103:721-5.

4. Cisco Public. CISCO Collaboration Work Practice Study. San Jose, California, USA: Cisco Public; 2013.

http://www.cisco.com/c/dam/en/us/solutions/collaboration/collaborationsales/cwps_full_report.pdf. Accessed 7 Jun 2016.

5. IEEE International Conference on Software Quality, Reliability and Security Companion; QRS; QRS-C; Information Assurance Workshop; Workshop on Information Assurance; Trustworthy Computing Workshop; Workshop on Trustworthy Computing; Workshop on Model-Based Verification and Validation; MVV; Workshop on Safety and Security in Cyber-Physical Systems; SSCPS Workshop; Workshop on Human and Social Aspect of Software Quality; HSASQ; Workshop on Software Engineering and Knowledge Management; SEKM Workshop. A Survey on Knowledge Management in Software Engineering: 3-5 August 2015, Vancouver, British Columbia, Canada : proceedings. Piscataway, NJ: IEEE; 2015.

6. Marshall C, Brereton P, Kitchenham B. Tools to support systematic reviews in software engineering. In: the 19th International Conference. p. 1-6. doi:10.1145/2745802.2745827.

7. Lu S, Elmaraghy W, Schuh G, Wilhelm R. A Scientific Foundation of Collaborative Engineering. CIRP Annals - Manufacturing Technology. 2007;56:605-34. doi:10.1016/j.cirp.2007.10.010.

8. Steinheider B. Supporting the co-operation of R\&D-teams in the product development process: Fraunhofer Institute for Industrial Engineering; 2001.

9. Patel H, Pettitt M, Wilson JR. Factors of collaborative working: A framework for a collaboration model. Applied Ergonomics. 2012;43:1-26. doi:10.1016/j.apergo.2011.04.009.

10. Pennington DD. Cross-Disciplinary Collaboration and Learning. Ecology and Society. 2008;13.

11. Müller P, Pasch F, Drewinski R, Hayka H. Kollaborative Produktentwicklung und digitale Werkzeuge: Defizite heute - Potenziale morgen. Berlin: Fraunhofer IPK; 2013. 